\title{
Zero-temperature resistive transition in Josephson-junction arrays at irrational frustration
}

\author{
Enzo Granato \\ Laboratório Associado de Sensores e Materiais, \\ Instituto Nacional de Pesquisas Espaciais, \\ 12245-970 São José dos Campos, SP Brazil
}

\begin{abstract}
We use a driven Monte Carlo dynamics in the phase representation to determine the linear resistivity and current-voltage scaling of a two-dimensional Josephson-junction array at an irrational flux quantum per plaquette. The results are consistent with a phase-coherence transition scenario where the critical temperature vanishes. The linear resistivity is nonzero at any finite temperatures but nonlinear behavior sets in at a temperature-dependent crossover current determined by the thermal critical exponent. From a dynamic scaling analysis we determine this critical exponent and the thermally activated behavior of the linear resistivity. The results are in agreement with earlier calculations using the resistively shunted-junction model for the dynamics of the array. The linear resistivity behavior is consistent with some experimental results on arrays of superconducting grains but not on wire networks, which we argue have been obtained in a current regime above the crossover current.

PACS numbers: 74.81.Fa, 74.25.Qt, 75.10.Nr
\end{abstract}

Most theoretical investigations of the vortex-glass phase in superconductors have considered model systems where there is a combined effect of quenched disorder and frustration ${ }^{1}$. However, in artificial Josephson-junction arrays, frustration without disorder can in principle be introduced by applying an external magnetic field on a perfect periodic array of weakly coupled superconducting grains $2,3,4$ and similarly on superconducting wire networks 5.6 . The frustration parameter $f$, the number of flux quantum per plaquette, is given by $f=\phi / \phi_{o}$, the ratio of the magnetic flux through a plaquette $\phi$ to the superconducting flux quantum $\phi_{o}=h c / 2 e$. It can be tuned by varying the strength of the external field. Frustration effects can be viewed as resulting from a competition between the underlying periodic pinning potential of the array and the natural periodicity of the vortex lattice ${ }^{7}$. At a rational value of $f$, the ground state is a commensurate pinned vortex lattice leading to discrete symmetries in addition to the continuous $U(1)$ symmetry of the superconducting order parameter. The resistive transition is only reasonably well understood for simple rational values of $f$.

At irrational values of $f$, the resistive behavior is much less understood since the vortex lattice is now incommensurate with the periodic array. In early Monte Carlo (MC) simulations ${ }^{8}$ the ground state was found to consist of a disordered vortex pattern lacking long range order which could be regarded as a some sort of vortexglass state without quenched disorder. Glassy-like behavior was indeed observed in these simulations suggesting a possible superconducting (vortex-glass) transition at finite temperatures. However, some arguments also suggested that the critical temperature should vanish ${ }^{7.9}$. Simulations of the current-voltage scaling using the resistively shunted-junction model for the dynamics of the array found that the behavior was consistent with an equilibrium resistive transition where the critical temperature vanishes 10 , similar to the resistive transition described by the the gauge-glass model in two dimensions ${ }^{1,11}$, but with different values for the correlation-length critical exponent $\nu$. The linear resistivity is nonzero at any finite temperatures but nonlinear behavior sets in at a crossover current with a temperature dependence determined by the exponent $\nu$. This zero-temperature transition leads to slow relaxation dynamics where the correlation length diverges as a power law and the relaxation time diverges exponentially as the temperature vanishes.

Simulations of the relaxation dynamics ${ }^{12}$ found a behavior analogous to relaxation in supercooled liquids with a characteristic dynamic crossover temperature rather than an equilibrium transition temperature, which is not inconsistent with the zero-temperature transition scenario. On the other hand, a systematic study by MC simulations 13 of a sequence of rational values of $f$ converging to the irrational frustration, using the vortex representation, found two phase transitions at finite temperatures, a vortex-order transition weakly dependent on $f$ and a vortex pinning transition at much lower temperatures varying with $f$, which should correspond to the resistive transition. These results are in qualitative agreement with MC simulations using the phase representation of the same mode ${ }^{14}$ but different ground states were found.

More recently, MC simulations for the the specific heat and relaxation dynamics found an intrinsic finitesize effect $\frac{15}{}$. The corresponding scaling analysis suggested a zero-temperature transition with a critical exponent $\nu$ consistent with the value obtained initially from current-voltage scaling 10 . However, a study of the low-temperature configurations for frustrations close the irrational value by $\mathrm{MC}$ simulations in the vortex representation 16 , find two phase transitions consistent with earlier work 13 .

On the experimental side, some results on arrays of su- 
perconducting grains at irrational frustration 2,3 are consistent with the scenario of the zero-temperature resistive transition but on wire networks ${ }^{5.6}$, resistivity scaling showed evidence of a transition at finite temperature. Recently, resistivity scaling suggesting a finite temperature transition was also observed in arrays of superconducting grains ${ }^{4}$.

In view of these conflicting results, it seems useful to further investigate the current-voltage scaling for the array at irrational frustration by studying both the nonlinear and linear resistivity with an improved method 17 taking into account the long relaxation times. In fact, as found recently, current-voltage scaling turned out to be quite reliable in determining the phase-coherence transition even for a model with quenched disorder, such as the three-dimensional XY-spin glass mode1 ${ }^{17}, 18$. The main question is therefore, if the array at irrational frustration displays an equilibrium phase-coherence transition at a nonzero critical temperature into a state with vanishing linear resistivity or its critical temperature vanishes and the linear resistivity is finite at nonzero temperatures.

In this work, we investigate the resistivity scaling of Josephson-junction arrays at a irrational frustration $f=$ $(3-\sqrt{5}) / 2$, a golden irrational, using a driven MC dynamics in the phase representation introduced recently 17 . The results are consistent with a phase-coherence transition scenario where the critical temperature vanishes, $T_{c}=0$. The linear resistivity is finite at nonzero temperatures but nonlinear behavior sets in at a temperaturedependent crossover current determined by the thermal critical exponent $\nu$. The results agree with earlier simulations using the resistively shunted-junction model for the dynamics of the array $\underline{10}$. However, with the present MC method we are able to reach much lower temperatures and current densities, improving the analysis of resistivity scaling and the estimate of the critical exponent $\nu$. We also argue that the finite-temperature transition found in resistivity measurements on wire networks ${ }^{5.6}$ have been obtained in a current regime above the crossover current.

We consider a two-dimensional Josephson-junction square array described by the Hamiltonian

$$
H=-J_{o} \sum_{<i j>} \cos \left(\theta_{i}-\theta_{j}-A_{i j}\right)-J \sum_{i}\left(\theta_{i}-\theta_{i+x}\right)
$$

The first term gives the Josephson-coupling energy between nearest neighbor grains where line integral of the vector potential $A_{i j}$ is constrained to $\sum_{i j} A_{i j}=2 \pi f$ around each plaquette. The second term represents the effects of an external driving current density $J$ applied in the $x$ direction. When $J \neq 0$, the total energy is unbounded and the system is out of equilibrium. The lowerenergy minima occur at phase differences $\theta_{i}-\theta_{i+x}$ which increases with time $t$, leading to a net phase slippage rate proportional to $\left\langle d\left(\theta_{i}-\theta_{i+x}\right) / d t\right\rangle$, corresponding to the voltage $V_{i, i+x}$. We take the frustration parameter $f$ equals an irrational number, $f=(3-\sqrt{5}) / 2$, related to the Golden Ratio $\Phi=(1+\sqrt{5}) / 2$ as $f=1-1 / \Phi$. In the numerical simulations we use periodic (fluctuating twist) boundary conditions on lattices of linear sizes $L$ and corresponding rational approximations $\Phi=F_{n+1} / F_{n}$, where $F_{n}$ are Fibonacci numbers $(13,21,34,55)$, with $L=F_{n}$.

To study the current-voltage scaling, we use a driven MC dynamics method ${ }^{17}$. The time dependence is obtained by identifying the MC time as the real time $t$ and we set the unit of time $d t=1$, corresponding to a complete MC pass through the lattice. Periodic (fluctuating twist) boundary conditions are used 19 . This boundary condition adds new dynamical variables, $u_{\alpha}(\alpha=x$ and $y$ ), corresponding to a uniform phase twist between nearest-neighbor sites along the principal axis directions $\hat{x}$ and $\hat{y}$. A MC step consists of an attempt to change the local phase $\theta_{i}$ and the phase twists $u_{\alpha}$ by fixed amounts, using the Metropolis algorithm. If the change in energy is $\Delta H$, the trial move is accepted with probability $\min \{1, \exp (-\Delta H / k T)\}$. The external current density $J$ in Eq. 1 biases these changes, leading to a net voltage (phase slippage rate) across the system given by

$$
V=\frac{1}{L} \frac{d}{d t} \sum_{j=1}^{L}\left(\theta_{1, j}-\theta_{L+1, j}-u_{x} L\right),
$$

in arbitrary units. The main advantage of this $\mathrm{MC}$ method compared with the Langevin dynamics used earlier ${ }^{10}$ is that in principle much longer time scales can be accessed which allows one to obtain reliable data at much lower temperatures and current densities. We have determined the electric field $E=V / L$ and nonlinear resistivity $\rho=E / J$ as a function of the driving current density $J$ for different temperatures $T$ and different system sizes. We used typically $2 \times 10^{5} \mathrm{MC}$ steps to reach the nonequilibrium steady state at finite current and equal time steps to perform time averages with and additional average over $4-6$ independent runs.

We have also determined the linear resistivity, $\rho_{L}=$ $\lim _{J->0} E / J$, from equilibrium MC simulations. As any transport coefficient, this quantity can be obtained from equilibrium fluctuations and therefore can be calculated in absence of an imposing driving current $(J=0)$. From Kubo formula, the linear resistivity (resistance in two dimensions) is given in terms of the equilibrium voltage autocorrelation as

$$
\rho_{L}=\frac{1}{2 T} \int d t\langle V(t) V(0)\rangle
$$

Since the total voltage $V$ is related to the phase difference across the system $\Delta \theta(t)$ by $V=d \Delta \theta(t) / d t$, we find more convenient to determine $\rho_{L}$ from the long-time equilibrium fluctuations $\frac{11}{11}$ of $\Delta \theta(t)$ as

$$
\rho_{L}=\frac{1}{2 T t}\left\langle(\Delta \theta(t)-\Delta \theta(0))^{2}\right\rangle,
$$

which is valid for sufficiently long times $t$. To insure that only equilibrium fluctuations are considered, the calculations were performed in two steps. First, simulations using the exchange MC method (parallel tempering) ${ }^{20}$ 


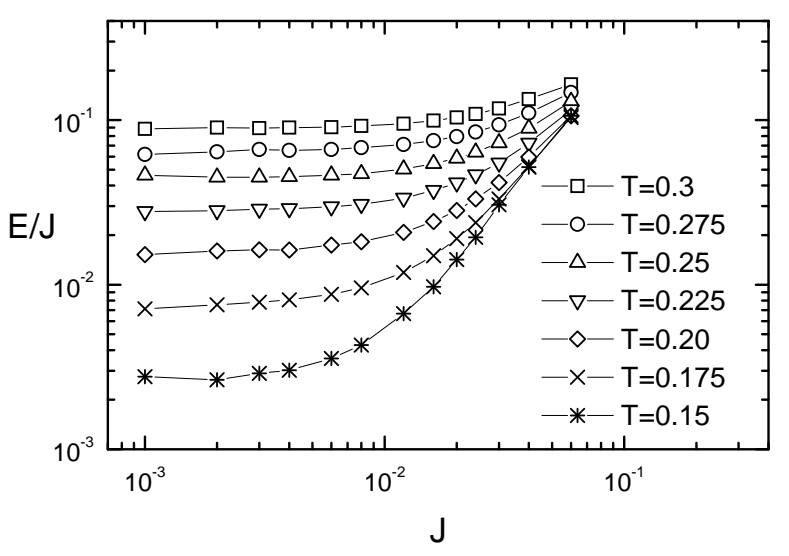

FIG. 1: Nonlinear resistivity $E / J$ at different temperatures $T$ for system size $L=55$.

were used to obtain equilibrium configurations of the systems at different temperatures 21 . This method is known to reduce significantly the critical slowing down near the transition allowing fully equilibration in finite small system sizes. These configurations were then used as initial states for the driven MC dynamics process described above, with $J=0$, in order to obtain the $\rho_{L}$. The initial states are similar to the low-temperature states obtained previously 13,16 including thermal excitations. In the parallel-tempering method ${ }^{20}$, many replicas of the system with different temperatures are simulated simultaneously and the corresponding configurations are allowed to be exchanged with a probability satisfying detailed balance. The equilibration time can be measured as the average number of $\mathrm{MC}$ steps required for each replica to travel over the whole temperature range. We used typically $4 \times 10^{6}$ (parallel tempering) MC steps for equilibration which is much larger than the estimated equilibration time in systems with up to 100 replicas. Subsequent MC simulations for the linear resistivity obtained from Eq. 4 were performed using $2 \times 10^{3}$ time averages for $2 \times 10^{5} \mathrm{MC}$ steps which is much larger than the equilibrium relaxation time.

Fig. 1a shows the nonlinear resistivity $E / J$ as a function of temperature for the largest system size. At small current densities $J$, the nonlinear resistivity $E / J$ tends to a constant value, corresponding to the linear resistivity $\rho_{L}$, which decreases rapidly with decreasing temperature. For increasing $J$, the resistivity cross over to a nonlinear behavior at a characteristic current density $J_{n l}$, which also decreases with decreasing temperature. To verify that the nonzero values approached at low currents in Fig. 1 correspond indeed to the linear resistivity $\rho_{L}$, we show in Fig. 2 the temperature dependence of $\rho_{L}$ obtained without current bias from Eq.(4) for different system sizes. $\rho_{L}$ decreases with system size but approaches nonzero values for the largest system size. These values are in agreement with the corresponding values at the lowest current in Fig. 1. Since the be-

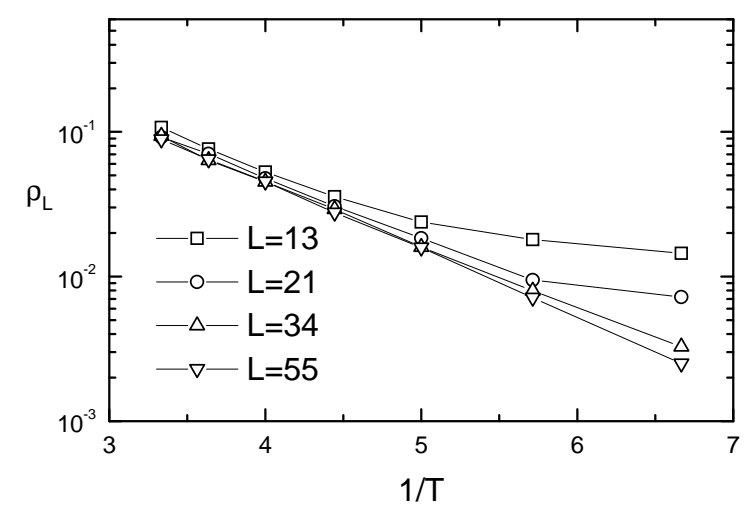

FIG. 2: Temperature dependence of the linear resistivity for different system sizes.

havior of the $\rho_{L}$ for the largest system size on the loglinear plot in Fig. 2 is a straight line, it indicates an activated Arrhenius behavior, where the linear resistivity decreases exponentially with the inverse of temperature with a temperature-independent energy barrier, estimated as $E_{b} \sim 1.07$. Such activated behavior suggests that the linear resistivity can be very small at low temperatures but nevertheless remains finite for all temperatures $T>0$ and therefore there is no resistive transition at finite temperatures. However, as will be described below, the system behaves as if a resistive transition occurs at zero temperature, corresponding to a phasecoherence transition where the critical temperature vanishes, $T_{c}=0$.

The behavior of the linear resistivity can be related to the equilibrium relaxation time for phase fluctuations. Since the voltage is the rate of change of the phase, a nonzero $\rho_{L}$ requires measurements over a time scale $\tau \propto 1 / \rho_{L}$, corresponding to the relaxation time for phase fluctuations. Thus, we expect that $\tau$ should also have an activated behavior, increasing exponentially with the inverse of temperature. To verify this behavior, we have in addition calculated the relaxation time $\tau$ for different temperatures from the autocorrelation function of phase fluctuations $C(t)$ as

$$
\tau=\frac{1}{C(0)^{2}} \int_{0}^{\infty} d t C(t)
$$

using MC simulations with $J=0$. The starting configurations were taken from equilibrium configurations obtained $^{21}$ with the parallel tempering MC method 20 . The results shown on the log-linear plot in Fig. 3 are indeed consistent with an activated behavior of $\tau$ with an energy barrier $E_{b}=1.18$ in reasonable agreement with the value obtained for the linear resistivity in Fig. 2.

The behavior in Figs. 1, 2 and 3 has the main features associated with a phase transition that only occurs at zero temperature, $T_{c}=0$, similar to the two-dimensional gauge glass model of disordered superconductors 1,11 . In this case the correlation length $\xi$ is finite for $T>0$ but it 
increases with decreasing temperature as $\xi \propto T^{-\nu}$, with $\nu$ a critical exponent. The divergent correlation length near the transition determines both the linear an nonlinear resistivity behavior leading to current-voltage scaling sufficiently close to the critical temperature and sufficiently small driving current. To understand in detail the behavior of the linear $\rho_{L}$ and nonlinear resistivity $\rho$ we need a scaling theory for the resistive behavior. If the data satisfy such scaling behavior for different driving currents and temperatures, the critical temperature and critical exponents of the underlying equilibrium transition at $J=0$ can then be determined from the best data collapse. A detailed scaling theory has been described in the context of the current-voltage characteristics of vortex-glass models 1 but the arguments should also apply to the present case. The basic assumption is the existence of a second order phase transition. Measurable quantities should then scale with the diverging correlation length $\xi \propto\left|T-T_{c}\right|^{-\nu}$ and relaxation time $\tau$ near the critical point. The nonlinear resistivity $E / J$ should then satisfy the scaling form ${ }^{1}$

$$
T \frac{E}{J} \tau=g_{ \pm}\left(\frac{J \xi}{T}\right)
$$

in two-dimensions, where $g_{ \pm}(x)$ is a scaling function. The + and - signs correspond to $T>T_{c}$ and $T<T_{c}$, respectively. If $T_{c} \neq 0$, then to satisfy such scaling form, the nonlinear resistivity curves on the log-log plot in Fig. 1 should have a positive curvature at small $J$, with $E / J$ decreasing with decreasing $J$ to a temperature dependent value for $T>T_{c}$ while for $T<T_{c}$, the curvature should be negative, with $E / J$ vanishing in the limit $J \rightarrow 0$. The data in Fig. 1 do not show a change in curvature even for the lowest temperature, already suggesting the possibility of a resistive transition at much lower temperatures or at $T_{c}=0$. However, a full scaling analysis of the data is required to show that a transition indeed occur with $T_{c}=0$. If $T_{c}=0$, then the correlation length $\xi \propto T^{-\nu}$ and the linear resistivity $\rho_{L}$ are both finite at $T>0$. One can then consider the behavior of the dimensionless ratio $E / J \rho_{L}$ which should satisfy the scaling form

$$
\frac{E}{J \rho_{L}}=g\left(\frac{J}{T^{1+\nu}}\right)
$$

where $g$ is a scaling function with $g(0)=1$. A crossover from linear behavior, when $g(x) \sim 1$, to nonlinear behavior, when $g(x)>>1$, occurs when $x \sim 1$ which leads to a characteristic current density at which nonlinear behavior sets in decreasing with temperatures as a power law, $J_{n l} \propto T / \xi \propto T^{1+\nu}$. The scaling form in Eq. (7) contains a single critical exponent $\nu$ and does not depend on the particular form assumed for the divergence of the relaxation time $\tau$. However, for sufficiently low temperatures, the relaxation process is expected to be thermally activated $\underline{1}$ with $\tau \propto \exp \left(E_{b} / k T\right)$. This corresponds formally to a dynamic exponent $z \rightarrow \infty$, if power-law behavior is assumed for the relaxation time $\tau \propto \xi^{z}$. From the scaling form of Eq.(6), the linear resistivity should scale as

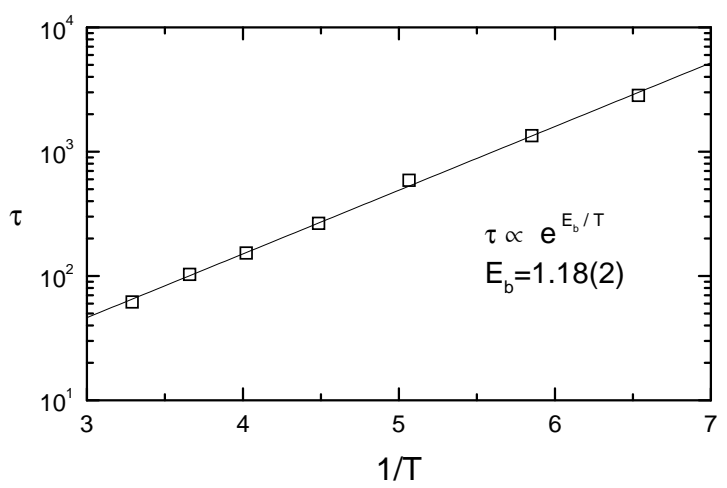

FIG. 3: Temperature dependence of the relaxation time $\tau$ of phase fluctuations for system size $L=55$.

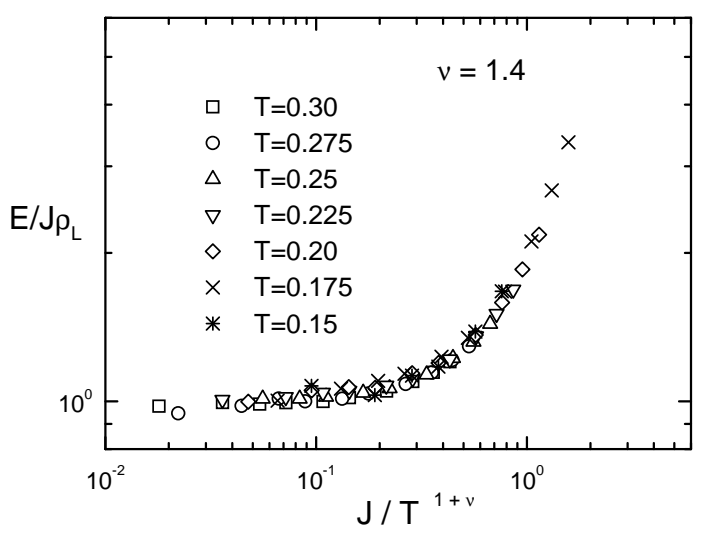

FIG. 4: Scaling plot of the nonlinear resistivity in Fig. 1 for $\nu=1.4$.

$\rho_{L} \propto 1 / \tau$ and therefore it is also expected to have an activated behavior, $\tau \propto \exp \left(-E_{b} / k T\right)$. In general, the energy barrier $E_{b}$ also scales with the correlation length as $E_{b} \propto \xi^{\psi}$, which leads to a temperature-dependent barrier $E_{b} \propto T^{-\psi \nu}$. A pure Arrhenius behavior corresponds to $\psi=0$. The behavior of the nonlinear and linear resistivity in Figs 1, 2 and the relaxation time in Fig. 3 are quite consistent with these predictions from the scaling theory of a zero-temperature transition.

If there is a zero-temperature transition, as suggested by the behaviors in Figs. 1, 2 and 3, then the data for the nonlinear resistivity should satisfy the scaling form of Eq.(7), if finite-size effects are negligible, and the best data collapse provides an estimate of the critical exponent $\nu$. We expect that finite-size effects are negligible for the largest system size $L=55$ in Fig. 1 since at this length scale the behavior of the linear resistivity is roughly independent of the size as can be seen from Fig. 2. Fig. 4 shows that indeed the data for the largest system size satisfy this scaling form with $\nu \sim 1.4 \pm 0.2$.

The nonlinear resistivity should also satisfy the expected finite-size behavior in smaller system sizes when 
the correlation length $\xi$ approaches the system size $L$. According to finite-size scaling, the scaling function in Eq. (7), should also depend on the dimensionless ratio $L / \xi$ and so to account for finite-size effects the nonlinear resistivity should satisfy the scaling form

$$
\frac{E}{J \rho_{L}}=\bar{g}\left(\frac{J}{T^{1+\nu}}, L^{1 / \nu} T\right)
$$

The scaling analysis of the whole nonlinear resistivity data is rather complicated in this case since the scaling function depends on two variables. To simplify the analysis 22 we first estimate the temperature and finite-size behavior of the crossover current density $J_{n l}$ where nonlinear behavior sets in as the value of $J$ where $E / J \rho_{L}=C$, a constant. Then, from Eq. (8), the finitesize behavior of $J_{n l}$ can be expressed in the scaling form

$$
J_{n l} L^{(1+\nu) / \nu}=\overline{\bar{g}}\left(L^{1 / \nu} T\right) .
$$

The best data collapse according to the scaling in Eq. (9) provides an alternative estimate of the critical exponent $\nu$. Fig. 5 shows that indeed the values of $J_{n l}$ for different system sizes and temperatures satisfy this scaling form with $\nu \sim 1.4$, in agreement with the estimate obtained for the largest system in Fig. 4 size using Eq. (7).

In addition to the standard finite-size effects, which occur when the correlation length is comparable to the system size, already taken into account in the scaling form of Eq. (8), there are also intrinsic finite-size effects 15 resulting from the rational approximations used for the irrational value of $f$. Since we use rational approximations $\Phi=F_{n+1} / F_{n}$, where $F_{n}$ are Fibonacci numbers $(13,21,34,55)$, with the system size set to $L=F_{n}$, this amounts essentially to have different values of the frustration, $f_{L}=1-1 / \Phi$, for different system sizes which will only converge to the correct value $f=(3-\sqrt{5}) / 2$ in the infinite-size limit. We have assumed that such effects are negligible in the above scaling analysis but they should affect our estimate of the critical exponent $\nu$. In principle, this intrinsic effect could be taken into account within the zero-temperature transition scenario by allowing for a size-dependent critical temperature $T_{c}(L)$ in the scaling analysis $\frac{15}{}$. Alternatively, we could regard it as a crossover from the critical behavior at the true irrational frustration (infinite-size limit) to a phase with an additional small frustration $\delta f=f_{L}-f$ which should act as a relevant perturbation. In this case, the scaling function in Eq. (7) should also depend on the dimensionless ratio $\xi^{2} \delta f$ and again a scaling analysis with more than one variable is required. However, our present numerical data is not sufficiently accurate to separate this effect from standard finite-size effects.

The present results for the linear and nonlinear resistivity of the array at irrational frustration obtained by the driven MC dynamics agree with earlier simulations of the current-voltage scaling using the resistively shuntedjunction model for the dynamics of the array $\stackrel{10}{\underline{10}}$, where a zero-temperature resistive transition was suggested and

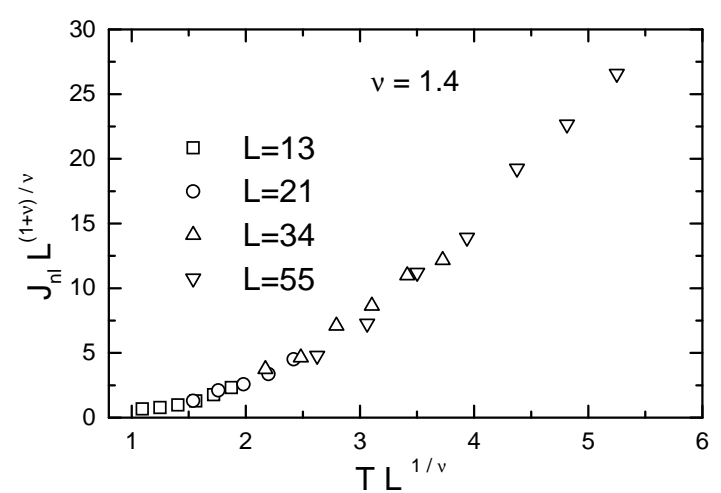

FIG. 5: Finite-size scaling plot of the crossover current density $J_{n l}$ with $\nu=1.4$, for different system sizes $L$.

the critical exponent was estimated as $\nu=0.9(2)$. Although the later model is expected to be a more realistic description for the dynamics of the array, the value of the static critical exponent $\nu$ should be the same for both models. In general, the dynamic exponent $z$ may depend on the particular dynamics but since the relaxation time $\tau$ is found to diverge exponentially for decreasing temperature it corresponds to $z \rightarrow \infty$ for both dynamics. The present estimate of $\nu=1.4(2)$, however, should be more reliable since it considers much lower temperatures and current densities and larger system size. Interestingly, similar behavior for the resistive transition has been found both numerically and experimentally for two-dimensional disordered superconductors in a magnetic field described as a gauge-glass mode $1^{1,11}$ but with different value for critical exponent $\nu \sim 2$. It should be noted however that the actual ground state at irrational frustration (without disorder) can be quite different, as the self similar structure which has already been $\operatorname{proposed}^{5.23}$. As would be expected, the different nature of ground state leads to the different values of the critical exponent $\nu$.

Although the above scaling analysis is consistent with a zero temperature transition, on pure numerical grounds the data in Figs 1 and 2 can not complete ruled out a vortex-order or a phase-coherence transition at temperatures much lower than $T=0.15$. In fact, phasecoherence transitions were found in MC simulations using the Coulomb-gas presentation 13 at temperatures as low as $T \sim 0.03$ for the sequence of rational approximations $f_{L}$ of the irrational $f$ but since they show considerable variation with $f_{L}$ it is not clear if it will remain nonzero in the large size limit. However, the lowest temperature in Figs 1 and 2 is already much smaller than the apparent freezing temperature $T_{f} \sim 0.25$ observed in earlier MC simulations ${ }^{-}$. Below $T_{f}$, a nonzero Edwards-Anderson order parameter $q(t)=<\vec{S}_{i}>^{2}$, was observed, where $\vec{S}=(\cos \theta, \sin \theta)$ and the average was taken over the simulation times $t$. Although this could suggest a diverging relaxation time $\tau \propto \int_{o}^{\infty} q(t) d t$ near a finite temperature $T_{c} \sim T_{f}$, such long relaxation time can also result from a 
zero-temperature transition $\left(T_{c}=0\right)$ as suggested by the above scaling analysis since in this case $\tau$ diverges exponentially with decreasing temperature, $\tau \propto \exp \left(E_{b} / k T\right)$, as shown in Fig. 3. For low enough temperatures, $\tau$ will eventually be larger than any simulation or experimental measuring time scale and an apparent (time dependent) freezing transition could occur depending on the particular dynamics and system size.

Some experimental results on arrays of superconducting grains at irrational frustration ${ }^{2,3}$ are consistent with the scenario of a zero-temperature resistive transition since even at the lowest temperatures a zero-resistance state was not observed in these experiments. On the other hand, current-voltage scaling analysis of experimental data on wire networks 5.6 was found to be consistent with a resistive transition at finite temperature. We note, however, that although the equilibrium behavior of wire networks can be described by the same model of Eq. 1, the nonlinear dynamical behavior may be quite different since the nodes of the network are connected by continuous superconducting wires, instead of weak links, leading to additional larger energy barriers for vortex motion, not included in the model, and consequently larger phase-coherence length $\xi$ and relaxation time $\tau$ when compared with weak links 24 . In this case, the characteristic crossover current to the linear resistivity regime at low temperatures due to thermal fluctuations, $J_{n l} \propto k T / \xi$, expected in the zero-temperature transition scenario, may only occur at current scales too small to be detected experimentally. Thus the resistive behavior is observed in a current regime at higher currents where it follows the mean-field theory result 25 where a vortexglass transition is possible at finite temperatures. However, the zero-temperature resistive could in principle be observed in specially prepared wire networks in the weak coupling regime where the additional energy barrier for vortex motion can be minimized ${ }^{26}$. Other effects, such as weak disorder, which is inevitably present in both experimental systems, should also be considered. It could provide a possible explanation for the finite-temperature resistive transition observed recently in arrays of superconducting grains $\underline{\underline{4}}$.

In conclusion, we have investigated the resistivity scaling of Josephson-junction arrays at a irrational frustra- tion using a driven $\mathrm{MC}$ dynamics $\frac{17}{7}$. The results are consistent with a phase-coherence transition scenario where the critical temperature vanishes, $T_{c}=0$. The linear resistivity is finite at nonzero temperatures but nonlinear behavior sets in at a crossover current determined by the thermal critical exponent $\nu$. The results agree with earlier simulations using the resistively shuntedjunction model for the dynamics of the array 10 and more recent MC simulations taking into account the intrinsic finite-size effect $\frac{15}{15}$. Although we have only studied the array at a particular value of irrational frustration, the golden mean, we believe that the conclusion of a zerotemperature phase-coherence transition should be valid for all irrationals but possibly with different values of the thermal critical exponent $\nu$. The main advantage of studying the golden mean value is that it is considered the farthest from the low-order rationals and so intrinsic finite-effects should be smaller. However, other irrational frustrations have also been studied numerically 15,23 and experimentally ${ }^{\underline{5}}$. The resistive behavior probes mainly the phase-coherence of the system and since we find that phase coherence is only attained at zero temperature, we can not address directly the question of the existence of a vortex-order transition at finite temperatures. In fact, vortex order does not require long-range phase coherence. Therefore, a vortex-order transition at zero temperature or at finite temperature is consistent with the present work. However, in view of the results for the supercooled relaxation ${ }^{12}$ suggesting an analogy to structural glasses such transition may be expected at finite temperature and in fact is consistent with MC simulations indicating a first-order vortex transition ${ }^{13,14,16}$. Thus, the interesting possibility arises where the array undergoes two transitions for decreasing temperature, a finite-resistance vortex-order transition at finite temperature and a superconducting transition only at zero temperature. This phase transition scenario and the predicted behavior of the linear and nonlinear resistivity provides an interesting experimental signature for a Josephson-junction array at irrational frustration.

This work was supported by FAPESP (grant 03/00541$0)$ and computer facilities from CENAPAD-SP.
${ }^{1}$ R.A. Hyman, M. Wallin, M.P.A. Fisher, S.M. Girvin, and A.P. Young, Phys. Rev. B 51, 15304 (1995); D.S. Fisher, M.P.A. Fisher, and D.A. Huse, Phys. Rev. B 43130 (1991).

2 J.P. Carini, Phys. Rev. B 38, 63 (1988).

3 H. S. J. Zant, H. A. Rijken, and J. E. Mooij, J. Low Temp. Phys. 82, 67 (1991).

4 I.C Baek, Y.J.Y Yu, and M.Y Choi, Phys. Rev. B 69, 172501 (2004).

${ }^{5}$ F.Yu, N.E. Israeloff, A.M. Goldman, and R. Bojko, Phys. Rev. Lett. 68, 2535 (1992).

${ }^{6}$ X.S. Ling, H.J. Lezec, M.J. Higgins, J.S. Tsai, J. Fujita, H.
Numata, Y. Nakamara, Y. Ochiai, C. Tang, P.M. Chaikin, and S. Bhattacharya, Phys. Rev. Lett. 76, 2989 (1996).

7 S. Teitel and C. Jayaprakash, Phys. Rev. Lett. 51, 1999 (1983).

8 T. Halsey, Phys. Rev. Lett. 55, 1018 (1985).

9 M.Y. Choi and D. Stroud, Phys. Rev. B 35, 7109 (1987).

10 E. Granato, Phys. Rev. B 54, 9655 (1996).

11 E. Granato, Phys. Rev. B 58, 11161 (1998).

12 B. Kim and S.J. Lee, Phys. Rev. Lett. 78, 3709 (1997).

13 P. Gupta, S. Teitel, M.J.P. Gingras, Phys. Rev. Lett. 80, 105 (1998). 
14 C. Denniston and C. Tang, Phys. Rev. B 60, 3163 (1999).

15 S.Y. Park, M.Y. Choi, B.J. Kim, G.S. Jeon, and J.S. Chung, Phys. Rev. Lett. 85, 3484 (2000).

16 S.J. Lee, J.R. Lee, and B. Kim, Phys. Rev. Lett. 88, 025701 (2002).

17 E. Granato, Phys. Rev. B 69, 144203 (2004).

${ }^{18}$ L.W. Lee and A.P. Young, Phys. Rev. Lett. 22, 227203 (2003).

19 W.M. Saslow, M. Gabay, and W.-M. Zhang, Phys. Rev. Lett. 68, 3627 (1992).

${ }^{20}$ K. Hukushima and K. Nemoto, J. Phys. Soc. Jpn. 65, 1604
(1996); E. Marinari and G. Parisi, Europhys. Lett. 19, 451 (1992).

21 E. Granato, unpublished.

22 C. Wengel and A.P. Young, Phys. Rev. B 56, 5918 (1997)

23 M.R. Kolahchi, Phys. Rev. B 59, 9569 (1999).

24 H. S. J. Zant, M.N. Webster, J. Romijn, and J.E. Mooij, Phys. Rev. B 42, 2647 (1990).

25 G. Parisi, J. Phys. A 27, 7555 (1994).

26 M. Giroud, O. Buisson, Y.Y. Wang, and B. Pannetier, J. Low Temp. Phys. 87, 683 (1992). 